\title{
Image hardenings by uv-, pulsed-electron beams and crosslinking agents
}

\author{
Hiroyuki HIRAOKA \\ IBM Almaden Rescarch Center \\ 650 Harry Road, San Jose, CA 95120-6099
}

For uv-hardening increase in oxygen concentration accelerates crosslinking rate than in air, and uv-exposure in nitrogen retards the reaction. Surfaces of photoresist images are photo-oxidized by intense uv-irradiation in air. However, the oxidized layer never extends over scveral hundred $\AA$ depth as measured by ESCA. Crosslinking extends far deeper, although it depends on the irradiating wavelength. The degree of crosslinkages is far densier near the surface than in the interior. A combination of heating and uv-exposure helps uv-hardening by spreading and acclerate crosslinking in resist images. Deep-uv light never reaches deeper beyond a few micron thick layer of photoresists and of polyamic acids. Thus, uv-hardening has a limit of its application. High aspect ratio images can be fabricated with photoimageable polyamic acid. However, in high temperature curing the images flow, yielding only low aspect ratio images of polyimides. Pulsed electron beam hardening can solve these problems of thick photoresists and of polyamic acids. For both type of images about 200 pulses of electron beams at $25 \mathrm{keV}$ are required, each pulse having a dose from $1 \mu \mathrm{C} / \mathrm{cm}^{2}$ to $10 \mu \mathrm{C} / \mathrm{cm}^{2}$ with a duration of about 100 nsec. Another method of resist image stabilization is chemical crosslinking, using various crosslinking agents. These image hardening processes are discussed in terms of advantages and disadvantages of the processes and chemical mechanisms involved. 


\section{J. Photopolym. Sci. Technol., Vol.2, No.3, 1989}

\section{Introduction}

Integrated circuit manufacturing with semiconductors, electronic packaging, and thin film magnetic recording head manufacturing, all have high temperature processes such as reactive ion etching, metal deposition, ion implantation and ion milling. Resist images have to withstand high temperature while keeping geometrical integrity. When we studied uv-hardening in 1980, the purpose was the prevention of thermal flow of resist images at about $240^{\circ} \mathrm{C}$. Although plasma resist image stabilization (PRIST) ${ }^{1}$ process was known at that time, we liked to invent a better one than PRIST. UV-hardening works quite well for 1 to $2 \mu \mathrm{m}$ thick resist images for a high temperature process such as chlorine-based RIE for aluminuim-copper etching. However, it has a limit to applicable thickness of resist images. With thick resist images, thicker than $3 \mu \mathrm{m}$, uv-hardening results in reticulation of surfaces because of large difference in a degree of crosslinking between surfaces and bottom layer. Although combination of photo-irradiation and heating is proposed to reduce this reticulation and enhance crosslinking, a different degrec of crosslinking in thick resist films invariably results in severe reticulation. Thick resist images are required for magnetic recording thin film head manufacturing and electronic packaging application.

Pulsed clectron beams don't have any restriction of resist thickness if the electron energy is about 25 $\mathrm{keV}$ and resist thickness does not exceed $10 \mu \mathrm{m}$. They provide fairly uniform crosslinkings throughout resist thickness. Pulsed electron beams can be generated in soft vacuum with a cold cathode, and they can be applicable to many applications, including thick resist image hardening and hardening of photo-imageable polyamic acid, which may flow in curing process. From equipment and process point of views, it does not present any greater complexity than photostabilization and PRIST.

Crosslinking agents can be added to conventional photoresists. After imagewise exposure and conventional development, heating or/and uv- exposure is used to initiate crosslinking, providing hard resist images. Conversely, resist images can be exposed to crosslinking agents such as a silazane either in gas phase or in liquid solution. The crosslinking appeared in resist, giving hard images, suitable to many applications. Various kind of hardening processes are discussed in terms of their features in applications and mechanisms involved.

\section{UV and pulsed electron beam hardenings}

\section{UV-hardening:}

A long uv-irradiation of photoresist images to a medium pressure mercury lamp resulted in image stabilization, preventing its thermal flow at high temperature. ${ }^{2}$ The medium pressure mercury lamp had a strong output at deep uv-region and also at $313 \mathrm{~nm}$ region. With or without a thin Pyrex glass filter the same image stabilization was obtained provided a longer exposure time was given for an experiment with a Pyrex filter with its cut-off wave length at $300 \mathrm{~nm}$. However, with a low pressure mercury lamp there is no significant output at $313 \mathrm{~nm}$. With this lamp no resist stabilization can be obtained with a Pyrex filter. ${ }^{3}$ The most effective wavelength for resist stabilization is reported to lie between 300 and $320 \mathrm{~nm} .{ }^{4} \mathrm{Wc}$ presented the data with AZ2400 photoresist,' but we concentrated our study on an IBM internal photoresist. At the same period, van Pelt mentioned deep uv-hardening effect without further elaboration. ${ }^{5}$

Our initial results on AZ2400 is shown in Fig. 1. Exposure of 10 min with deep uv-output of 1 $\mathrm{mW} / \mathrm{cm}^{2}$ was sufficient enough to prevent thermal flow of resist images at $155^{\circ} \mathrm{C}$. A longer exposure time and higher uv-dose were required for a higher temperature stabilization; for example, $3 \mathrm{~J} / \mathrm{cm}^{2}$ for $240^{\circ} \mathrm{C}$ stabilization. Since this work, many decp uv-lamps are developed for resist image stabilization, such as a 


\section{(a)}

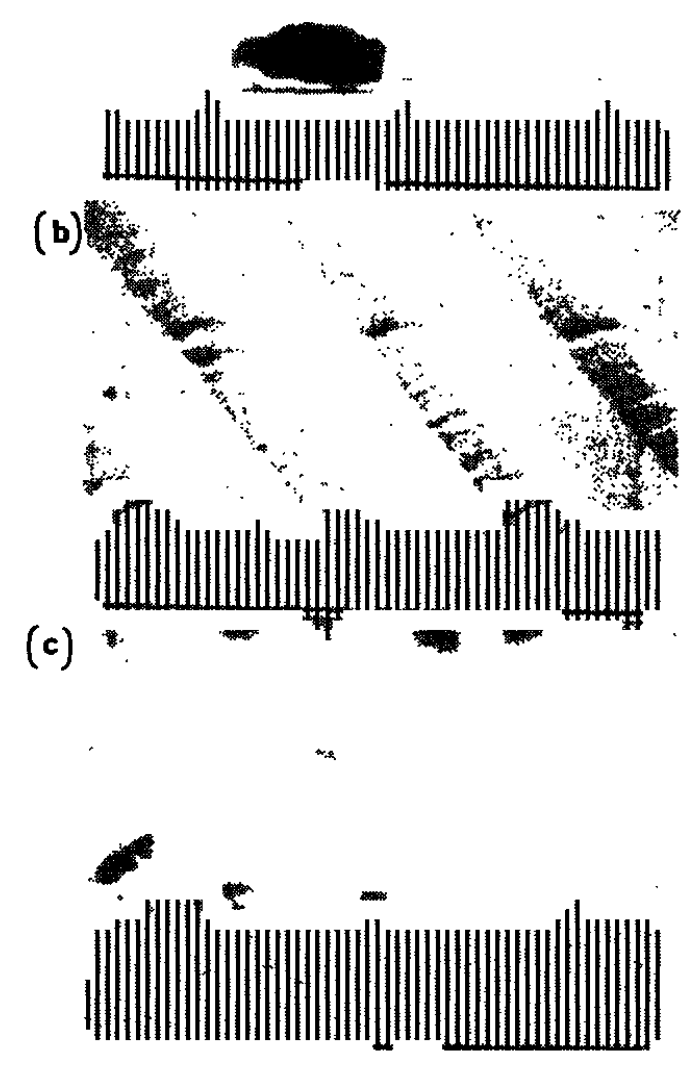

Figure 1 UV-hardening of AZ2400 patterns: (a) original patterns, (b) $155^{\circ} \mathrm{C}$ heating for $30 \mathrm{~min}$, (c) $155^{\circ} \mathrm{C}$ heating for $30 \mathrm{~min}$ after 10 min uv-exposure.

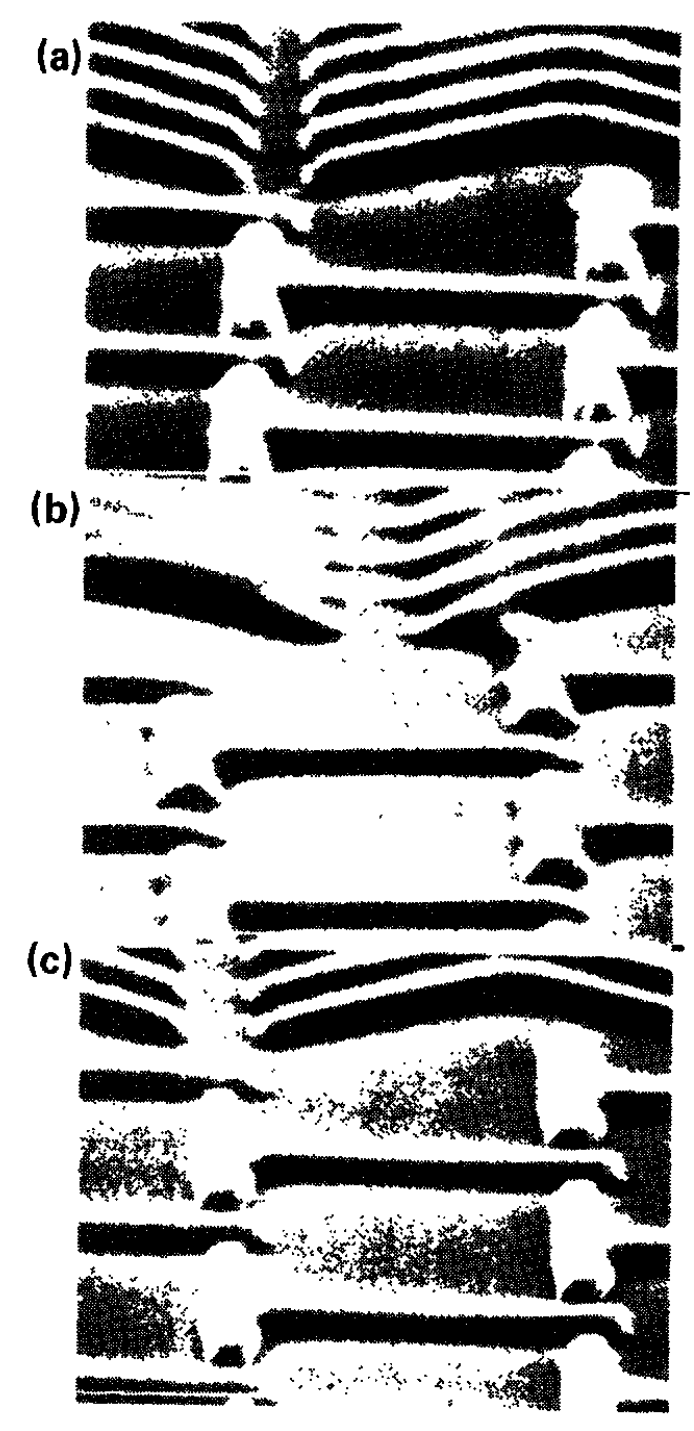

Figure 2 Poly(p-vinylphenol)-based negative photoresist: (a) original patterns, (b) images heated at $300^{\circ} \mathrm{C}$ for $30 \mathrm{~min},(\mathrm{c})$ the same as (b) except $1 \mathrm{hr}$ uv-exposure in air before heating.

microwave-driven lamp with deep uv-output of $800 \mathrm{~mW} / \mathrm{cm}^{2},{ }^{6}$ a low pressure mercury lamp with $254-\mathrm{nm}$ output of $40 \mathrm{~mW} / \mathrm{cm}^{27}$ and so forth. With intense uv-irradiation, the resist images are heated to a high temperature unless a wafer holder can control and regulate the temperature. Too rapid heating during uv-irradiation results in rounding of resist images. However, controlled heating in either post-bake or during uv-exposure helps crosslinking considerably. The stabilization passes through four distinct phases, depending on the thickness of uv-hardened skin of the resist. ${ }^{8}$ In the final phase with the largest uv-dose the geometrial dimension of resist images is maintained to the optimal degree. By a controlled heating at least $35 \%$ acceleration of crosslinking to attain the final phase is reported. 9 
$U V / B a k e^{8}$ process is a combination of uv-irradiation and heating process in a ramp mode, so that crosslinking reactions inside resist images spread as much as possible. UV-photostabilization alone is insufficient for the resist to withstand such processes as plasma etching of aluminum with copper. For such an etching, hardbake after uv-hardening or UV/Bake process is required. The minimum exposure dose for $1.1 \mu \mathrm{m}$ thick $\mathrm{KTI}-820$ is reported $0.7 \mathrm{~J} / \mathrm{cm}^{2}$ for resist on aluminum/copper and $0.9 \mathrm{~J} / \mathrm{cm}^{2}$ for resist on silicon dioxide..$^{10}$ Poly(vinylphenol)-based resists are also demonstrated to show uv-hardening effect, as shown in Figure 2." These resists showed higher temperature stability with the same uv-dose than novolac-based photoresists.

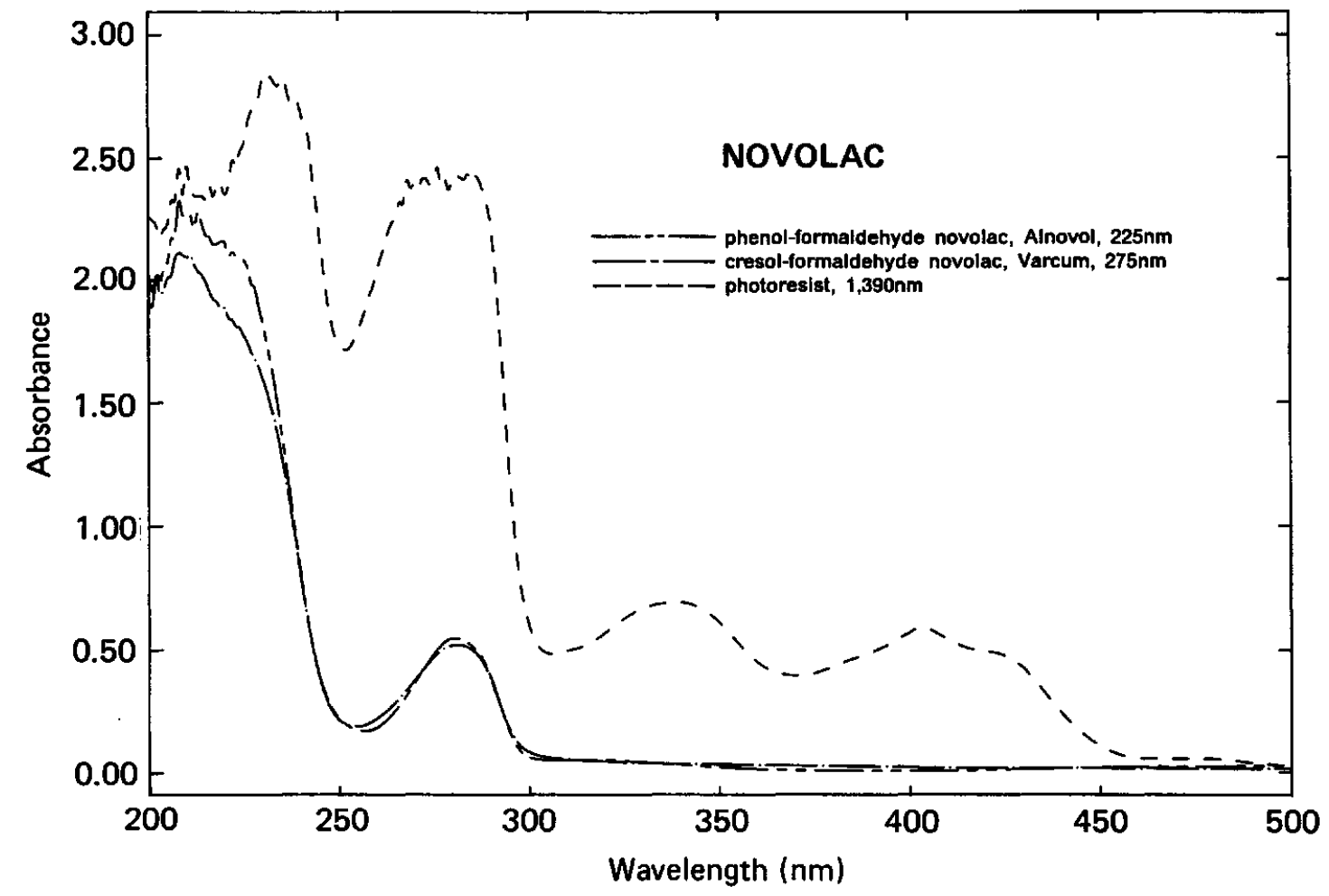

Figure 3 UV-absorption of novolac resin and novolac-based photoresist.

The major problem of uv-hardening arises when it is applied to a thick resist layer. As shown in Figure 3, photoresist films have strong uv-absorption below $300 \mathrm{~nm}$ wavelength. With resist films thicker than $3 \mu \mathrm{m}$ uv-induced crosslinking reactions take place only in upper surface layers, but not in the bottom layer, because no uv-light reaches there. A large difference in a crosslinking degree in the upper and lower layers result in reticulation after heating at high temperature, shown in Figure 4(c). Fine line images, however, don't show reticulation because uv-light illuminates the bottom layer from side-walls, as shown.

\section{Pulsed electron beam hardening:}

Clearly uv-hardening has some limit in its application to thick resist films. We are very much interested in geometrical integrity of thick resist images at high temperature. One way to overcome this limit is to use pulsed clectron beam hardening, as described here. The other method may be the use of chemical crosslinking agents which will be discussed next.

The application of pulsed electron beams for hardening is not limited by thickness of resist films nor by a degree of uv-absorption. This feature make it possible to apply to hardening of thick resist films and polyamic acid images, to which uv-hardening is not applicable. 
(a) No treatment, heated at $250^{\circ} \mathrm{C}$ for $10 \mathrm{~min}$, $x 1,000$ magnification, $35 \mu \mathrm{m}$ resist thickness
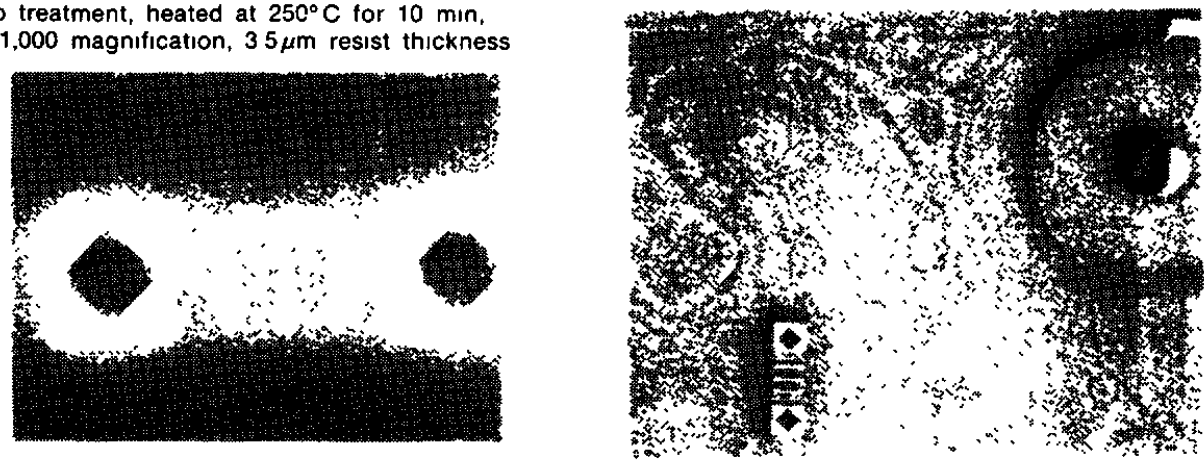

(b) Pulsed e-beam exposure at $25 \mathrm{KeV}, 200$ pulses $2 \times 10^{-3} \mathrm{C} / \mathrm{cm}^{2}$, heated at $250^{\circ} \mathrm{C}$ for $10 \mathrm{~min}$. $\times 1,000$
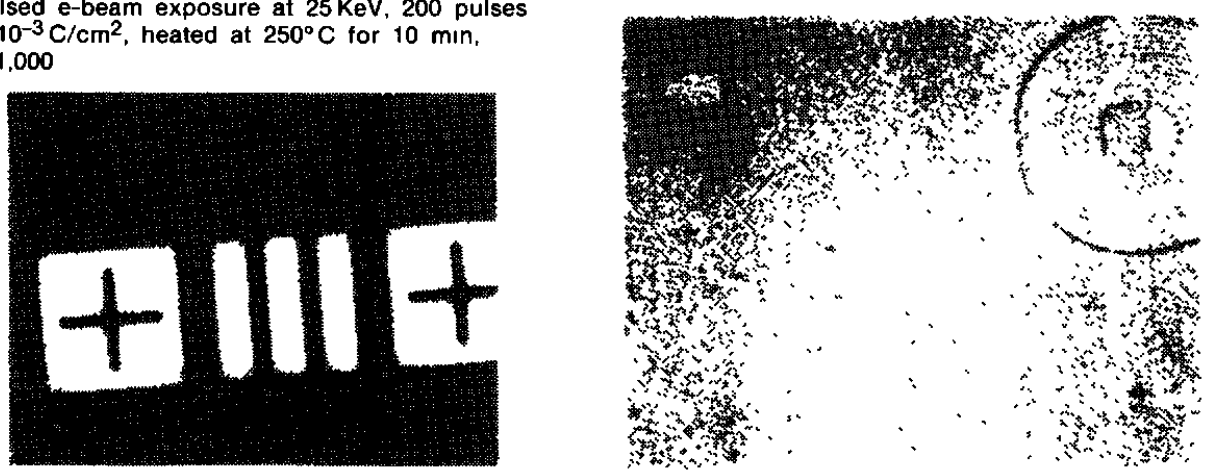

(c) Deep UV-exposure at 75 to $100^{\circ} \mathrm{C}$ for $75 \mathrm{~min}$ $6 \mathrm{~J} / \mathrm{cm}^{2}$, heated at $250^{\circ} \mathrm{C}$ for $10 \mathrm{~min}, \times 1,000$ magnification
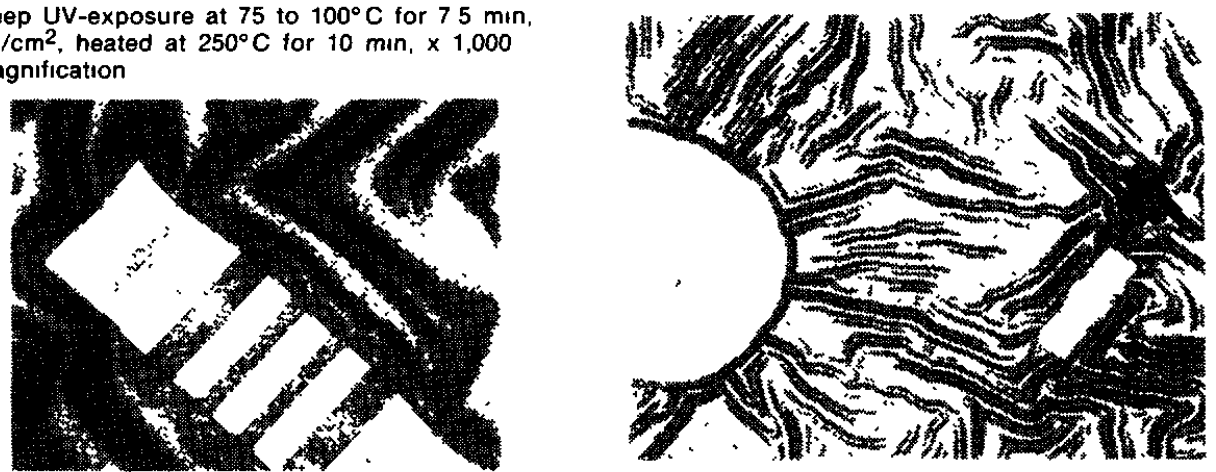

Figure 4 Comparison of uv-hardening and pulsed electron beam hardening of $3.5 \mu \mathrm{m}$ thick AZ-1375 images; note no reticulation of fine line images in uv-hardening because of side wall irradiation.

A schematic view of a pulsed electron beam hardening apparatus is shown in Figure $5 .^{12}$ Because it uses a flat cold cathode, different from a hot filament as an electron source, it operates in soft vacuum, not requiring a high vacuum. This is an important feature because hardening process generates gaseous products. Electron beam dose per pulse at $25 \mathrm{keV}$ ranges from $1 \mu \mathrm{C} / \mathrm{cm}^{2}$ to $10 \mu \mathrm{C} / \mathrm{cm}^{2}$. Hardening effect appears at about 200 pulses with $3.5 \mu \mathrm{m}$ thick photoresist films, as shown in Figure $4(\mathrm{~b})$. Heating at $250^{\circ} \mathrm{C}$ did not cause any reticulation in both large area and fine line images, while in the same heating condition photostabilized resist films showed severe reticulation in large imaged areas.

Several photo-imageable polyamic acids are available now. They provide good aspect ratio images before curing. However, the images flow in curing process, resulting in sloped wall angles of polyimide patterns. Polyamic acid (PMDA-ODA) images were fabricated by direct electron beam exposure through 


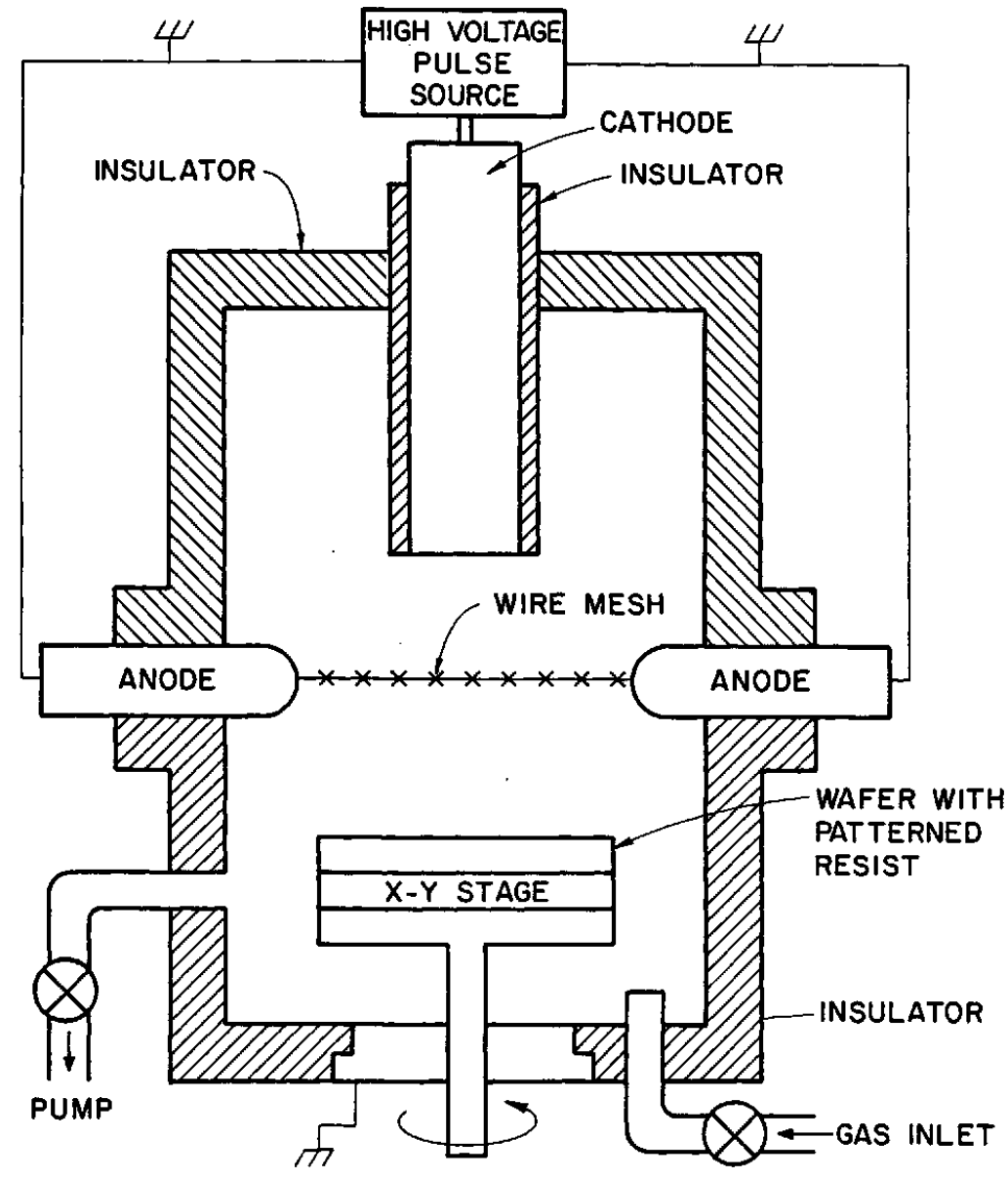

Figure 5 Pulsed electron beam cxposure apparatus.

Table 1 Polyamic acid (PMDA-ODA) image hardening by pulsed electron beams at $28 \mathrm{kV}$ in 25 mTorr air.

\begin{tabular}{|c|c|c|c|c|}
\hline \multirow[t]{2}{*}{$\begin{array}{l}\text { Hardening pulses, } \\
\text { No. }\end{array}$} & \multicolumn{2}{|c|}{$\begin{array}{l}\text { Wall angles, } \\
\text { degree }\end{array}$} & \multicolumn{2}{|c|}{$\begin{array}{l}\text { Thickness } \\
\mu \mathrm{m}\end{array}$} \\
\hline & Before & After & Before & After \\
\hline zero & 123 & 138 & 0.54 & 0.43 \\
\hline 125 & 120 & 133 & 0.64 & 0.46 \\
\hline 250 & 124 & 125 & 0.64 & 0.57 \\
\hline 500 & 127 & 129 & 0.50 & 0.50 \\
\hline
\end{tabular}

a stencil mask, taking 65 pulses of electron beams. Image developments were carried out by dipping wafers into NMP, 1-methyl-2-pyrrolidinone for $1 \mathrm{~min}$. Hardening was carried out by $28 \mathrm{kV}$ electron beams in 25 mTorr air with varied numbers of pulses. Hardened and unhardened samples were heated to $350^{\circ} \mathrm{C}$ for 30 min, cleaved and were examined by a SEM. As shown in Table 1, on-set of hardening is estimated around 200 pulses, corresponding to $2 \times 10^{-3} \mathrm{C} / \mathrm{cm}^{2}$. Thickness loss of unhardened images is clearly seen, while hardened patterns showed only a minimal thickness loss. Clearly electron beam exposures induced crosslinking and hardened the images in curing process, in the same way in uv-hardening of photoresist images. 


\section{Mechanistic discussions:}

Several different kinds of crosslinking reactions contribute to uv-hardening. Phenol-formaldehyde novolac resin is known to undergo oxidative coupling, crosslinking reaction when heated above $150^{\circ} \mathrm{C}$, as shown:
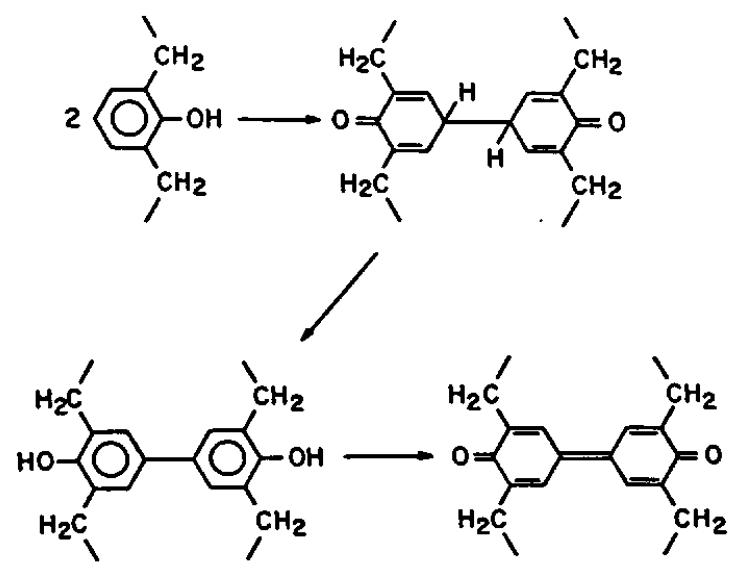

Thermal crosslinking or slow heating to a high temperature alone does not prevent image flow. Photo-induced crosslinkings of several kinds are required for effective uv-hardening by preventing initial rounding of resist images. UV-irradiation alone can cause similar oxidative coupling reaction to thermal one described above. Besides, linkage formations with ester and bisphenol moieties between photoproducts of DNQ and novolac chains take place, shown in the following:13<smiles>[R]Cc1cccc2c1C=CC(=[N+]=[N-])C2=O</smiles><smiles>CC1CC1C</smiles><smiles></smiles><smiles>[R]c1cccc2c1C=CC2C(=O)Oc1c(C)cccc1CC</smiles><smiles>CCc1cc(-c2ccc3c(PC)cccc3c2O)c(O)c(CC)c1C</smiles>

Our ESCA study of uv-hardened resist films showed photo-oxidation of resist surfaces, as shown by increasing oxygen contents and by new formations of carbonyl carbons and carbons with hydroxyl group in ESCA data of Figure 6 and Figure 7,respectively. The photo-oxidized layer extended only several hundred A. However, it is known that increase in oxygen concentration accelerates crosslinking rate for uv-hardening than in air, and that uv-exposure in nitrogen retards the reaction. The surface photo-oxidation helps to form hard skins of resist images.

In pulsed electron beam hardening no oxidized surface layer appeared. Its hardening function solely 


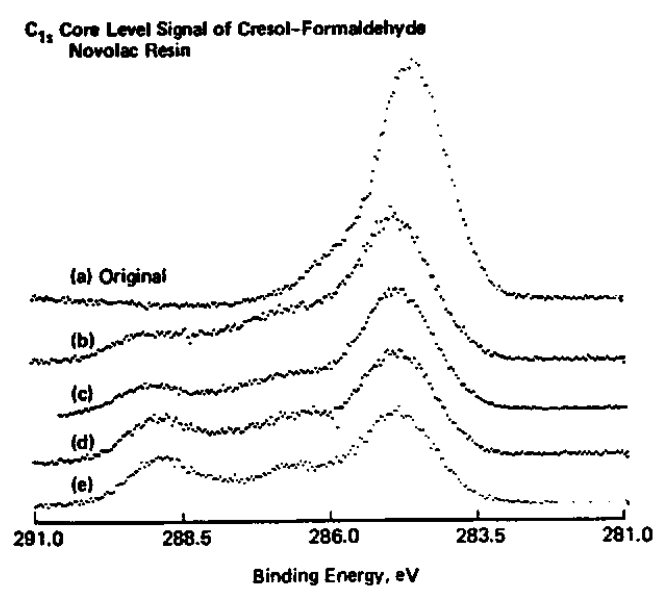

Figure 6 Change of the carbon core level signal as a function of uv-cxposure $(1 \mathrm{~mW} /$ $\mathrm{cm}^{2}$ at $254 \mathrm{~nm}$ ); (a) $0 \mathrm{hr}$, (b) $0.5 \mathrm{hr}$, (c) $1 \mathrm{hr}$, (d) $1.5 \mathrm{hr}$, (e) $4 \mathrm{hr}$

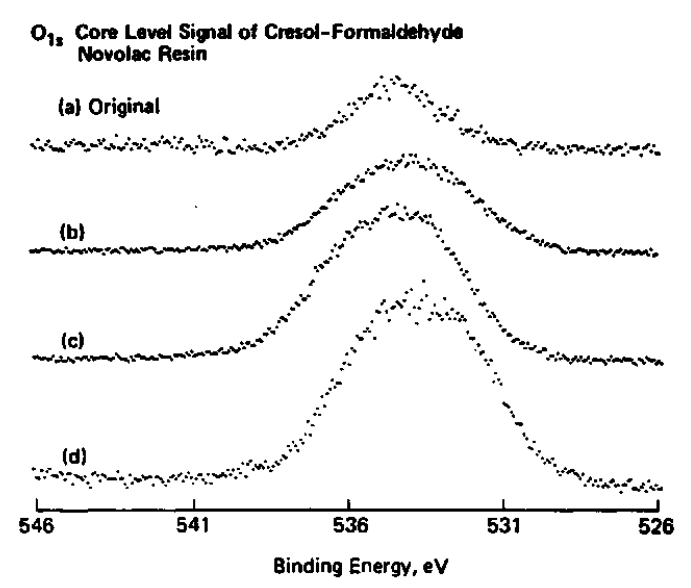

Figure 7 Change of the oxygen core level signal corresponding to Figure 6.

depended on extended crosslinkings in resist films. The major reaction of DNQ-novolac resists under a low dose of clectron beams is the same as in photo-decomposition. However, under high electron doses many other reactions leading to crosslinkings of novolac chains appear. With $25 \mathrm{keV}$ electron energy deposition is fairly uniform across thick resist films. Because of uniformly crosslinked network, no reticulation appeared after heating.

\section{Hardening by crosslinking agents}

Hardening can be accomplished by addition of crosslinking agents, which can be activated by photo-generated acid, heat or uv-light. Photoresist images which contain a melamine or an aromatic bisazide flow at high temperature before images are hardened. Once these images are crosslinked, they are very hard.

Certain crosslinking agents react before resist images flow. With crosslikned network, resist images maintain its geometrical dimension at high temperatures as high as $350^{\circ} \mathrm{C}$. Resist images are exposed to crosslinking agents in either vapor phase like $S A B R E$ system, ${ }^{14}$ or in liquid phase using various chemical reagents which include silazanes, ${ }^{15}$ alkyl magnesium and alkyl aluminum. ${ }^{16}$ Although these processes present additional chemical reactions which may not be favored in some cases, they provide additional advantage of enhanced oxygen reactive ion etch (RIE) resistance with silicon containing compounds, and enhanced halogen RIE resistance with magnesium and aluminum-containing compounds besides hardening of resist images. Figure 8 shows examples of typical surface hardenings of $3.5 \mu$ m thick AZ1375 images by chemical reagents such as dialkyl magnesium (c) and aluminum trichloride (d) in comparison with uv-hardening (b). With these chemical reagents hard skins are formed on resist surfaces, which prevented thermal flow with very little reticulation.

\section{Acknowledgments}

The study on pulsed electron beam hardening was carried out in collaboration with Dr. J. Krishnaswamy and Prof. G.J. Collins of Colorado State University. 


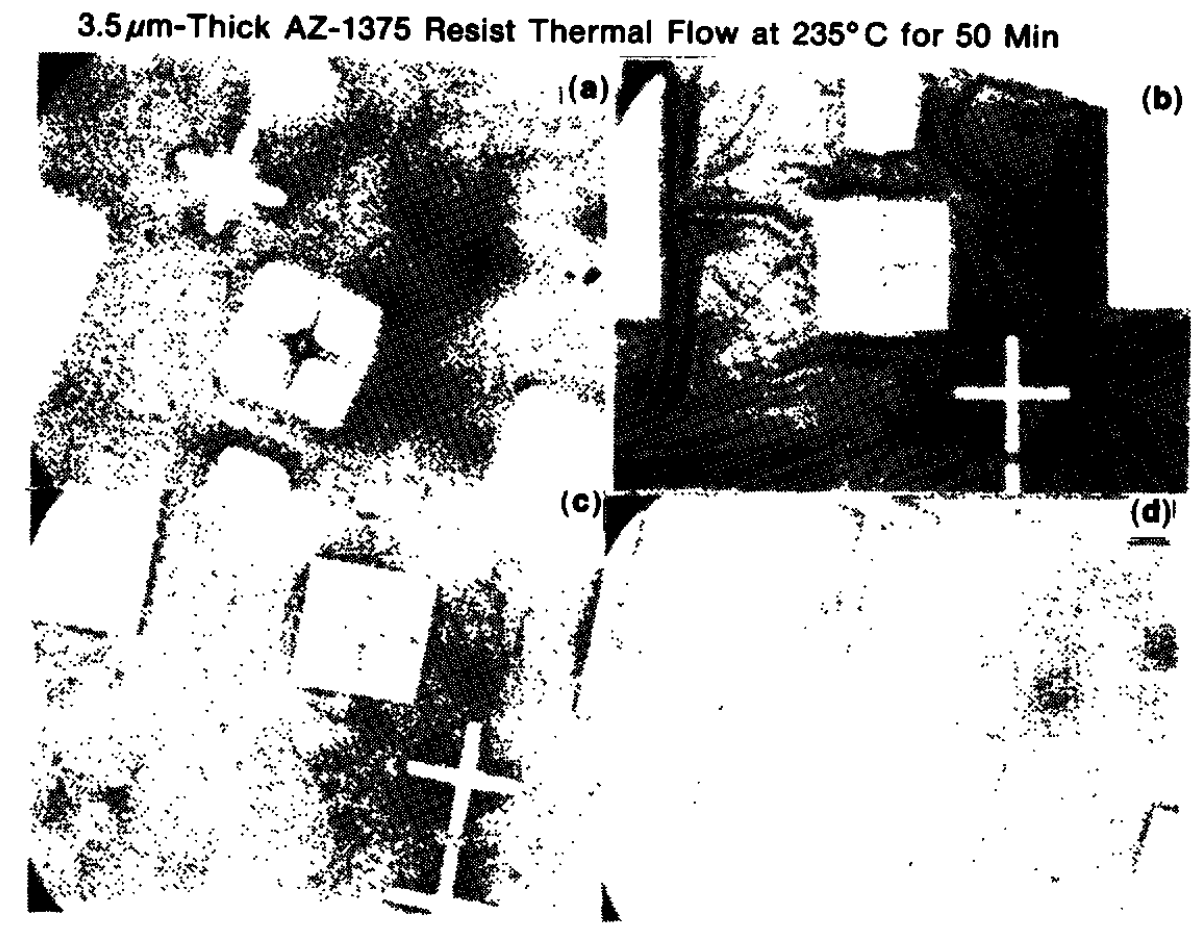

(b)

Figure 8

Comparison of (b) uv-hardening with chemical treatments:

(a) no treatment,

(c) dialkylmagnesium,

(d) $\mathrm{AlCl}_{3}$

\section{References}

1. W.H.-L. Ma, United States Patent No. 4,187,331.

2. II. Hiraoka, J. Pacansky, I6th Sym. Electron, Ion and Photon Beam Technology, May 27, 1981; J. Electrochem. Soc., 128, 2645 (1981); J. Vac. Sci. Technol., 19, 1132 (1981).

3. R. Allen, M. Foster, Y.-T. Yen, J. Electrochem. Soc., 129, 1379 (1982).

4. E. Spicrtz, F. Vollenbroek, Microcircuit Eng., 68-1 (1984).

5. P. van Pelt, SPIE Semiconductor Microlithography VI, Vol. 275, 150 (1981).

6. Microlite Series photostabilizer, Fusion semiconductor system, Rockville, MD20855.

7. UV Resist Stabilizer, UVP Inc., San Gabriel, CA91778.

8. J.C. Matthews, J.I. Willmott, Jr., SPIE Symp. Optical Microlithography III, Vol. 470, 194 (1984).

9. W.T. Babie, P.W. Klymko, IBM Tech. Discl. Bull. 27, 347 (1984).

10. Veeco Process Application Note \#109 March 1985, Veeco Instruments Inc., Plainview, NY11803.

11. H. Hiraoka, L.W. Welsh, Jr., The Tenth Intl. Conf. Electron and Ion Beam Sci. \& Technol., Vol. 83, pp.171-178 (1983). The Electrochem. Soc., Washington, D.C..

12. H. Hiraoka, Microelectronic Eng., 6, 407 (1987) ;H. Hiraoka, J. Lee, W. Hinsberg, J. Krishnaswamy, L. Li, G.J. Collins, The Electrochem. Soc. Meeting, Paper \#476, I Ionolulu, Hawai, October 1987; J. Krishnaswamy, L. Li, G.J. Collins, H. Iliraoka, M.A. Caolo, J. Mater. Res., 3(6), 1259 (1988).

13. A. Knop, W. Scheib, "Chemistry and Applications of Phenolic Resins," Springer-Verlag, New York, 1979.

14. M. Koshiba, M. Murata, M. Matsui, Y. Harita, SPIE Symp. Adv. Resist Technol. \& Processing V, Vol. 920,364 (1988).

15. W.C. McColgin, R.C. Daly, J. Jech, Jr., T.B. Brust, SPIE Symp. Adv. Resist Technol. Processing V, Vol. 920,260 (1988).

16. I. Hiraoka, to be presented at the 33rd Intl. Symp. Filectron, Ion, and Photon Beams, May 30-June 2, 1989, Monterey, California.

17. II. Iliraoka, SPIE Symp. Adv. Resist Technol. Processing IV, Vol. 77I, 174 (1987). 